\title{
Scalp Arteriovenous Fistula With Intracranial Communication
}

\author{
Stephen Hemperly, DO; Richard McClain, MD; Nektarios Lountzis, MD; Stephen M. Purcell, DO
}

\section{PRACTICE POINTS}

- Scalp arteriovenous fistulas may be traumatic or spontaneous and present as either an innocuouslooking scalp nodule or as a progressively enlarging pulsatile mass on the scalp.

- Clinical detection followed by appropriate imaging and referral to neurosurgery or interventional neuroradiology is vital to patient safety.

To the Editor:

A 71-year-old man presented with a nodule on the vertex of the scalp of 1 year's duration. The lesion had become soft and tender during the week prior to presentation. He noted that he was experiencing headaches and a buzzing sound in his head. He denied all other neurologic symptoms. The patient was given amoxicillin from a primary care physician and was referred to our institution for evaluation of a presumed inflamed cyst.

The patient's medical history included an intracranial arteriovenous fistula (AVF) treated with endovascular embolization 1 year prior to presentation, 2 substantial falls in childhood with head trauma and loss of consciousness, essential hypertension, and an aortic aneurysm. His medications included amlodipine, lisinopril, amoxicillin, a multivitamin, and grape seed extract.

Physical examination revealed a 2 - $\mathrm{cm}$, pink, somewhat rubbery, subcutaneous, nonmobile nodule on the vertex of the scalp (Figure 1). The lesion was not consistent with a common pilar cyst, and an excisional biopsy was performed to exclude malignancy. Upon superficial incision, the lesion bled moderately, and the procedure was immediately discontinued. Hemostasis was obtained, and the patient was sent for ultrasonography of the lesion.

Ultrasonography demonstrated a small hypoechoic nodule measuring up to $0.5 \mathrm{~cm}$ containing a tangle of vessels in the subcutaneous soft tissue corresponding to the palpable abnormality. A cerebral angiogram demonstrated a dural AVF of the superior sagittal sinus with multifocal supply that connected with this scalp nodule (Figure 2). The patient was treated by interventional neuroradiology with endovascular embolization, which resulted in complete resolution of the scalp nodule.

Scalp arteriovenous fistulas (S-AVFs) are characterized by abnormal connections between supplying arteries and

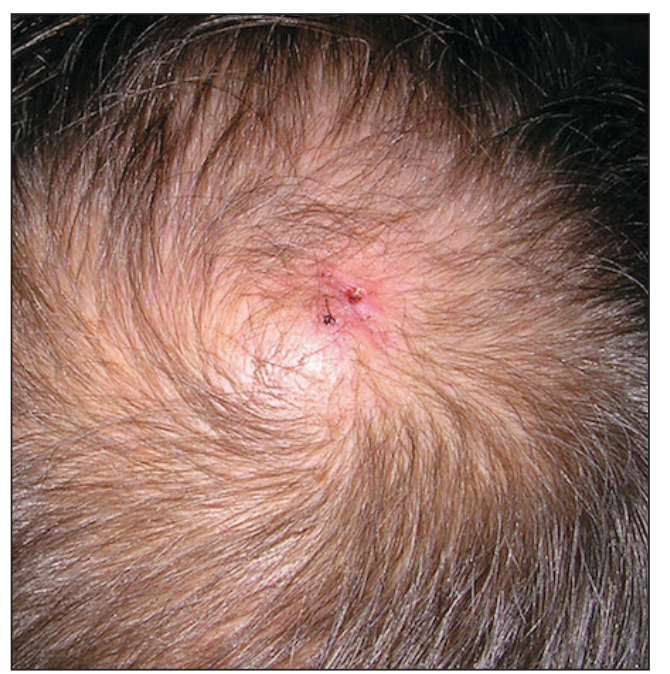

FIGURE 1. An arteriovenous fistula on the vertex of the scalp several weeks after the lesion was superficially incised for a biopsy.

Dr. Hemperly is from Lehigh Valley Health Network, Allentown, Pennsylvania. Drs. McClain, Lountzis, and Purcell are from Advanced Dermatology Associates, Ltd, Allentown.

The authors report no conflict of interest.

Correspondence: Stephen Hemperly, DO, Lehigh Valley Health Network, Dermatology Residency Program, 1259 S Cedar Crest Blvd, Allentown, PA 18103 (stephen.hemperly@gmail.com).

doi:10.12788/cutis.0121 


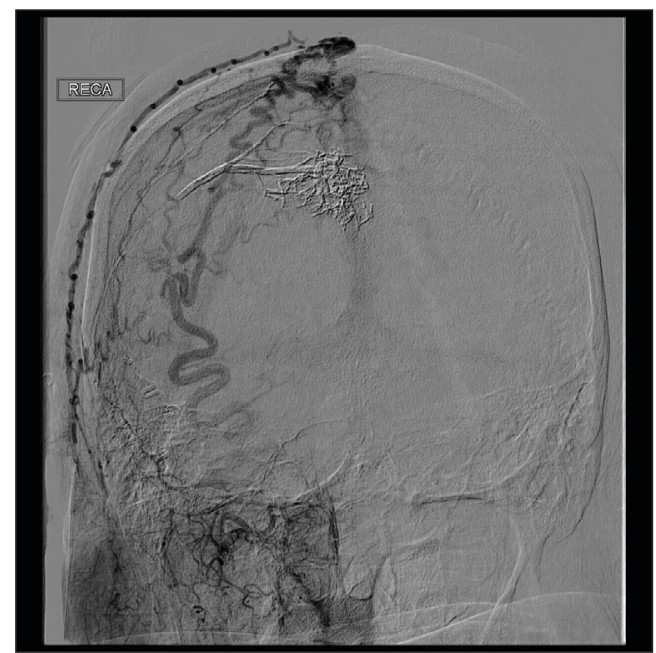

FIGURE 2. Cerebral angiogram demonstrated a dural arteriovenous fistula of the superior sagittal sinus with multifocal supply that connected with this scalp nodule.

draining veins in the subcutaneous plane of the scalp. ${ }^{1,2}$ The veins of an S-AVF undergo progressive aneurysmal dilatation from abnormal hemodynamics. ${ }^{1-3}$ Scalp arteriovenous fistulas are rare and may present as either an innocuous-looking scalp nodule or a progressively enlarging pulsatile mass on the scalp. ${ }^{2-4}$ Associated symptoms often include headache, local pain, bruits, tinnitus, and thrill. ${ }^{1,3,4}$ Recurrent hemorrhage, scalp necrosis, congestive heart failure, epilepsy, mental retardation, and intracranial ischemia also may occur. ${ }^{4}$

Scalp AVFs may occur with or without intracranial communication. ${ }^{4}$ Spontaneous S-AVFs with intracranial communication are uncommon, and their etiology is unclear. They may form as congenital malformations or may be idiopathic. Factors increasing circulation through the S-AVF such as trauma, pregnancy, hormonal changes, and inflammation prompt the development of symptoms. ${ }^{4}$ Scalp AVFs also may be caused by trauma. ${ }^{3}$ Scalp AVFs without intracranial communication have been reported following hair transplantation. ${ }^{1}$ Scalp AVFs with intracranial communication have been reported months to years after skull fracture or craniotomy. ${ }^{2}$ True spontaneous $\mathrm{S}$-AVFs are difficult to differentiate from traumatic S-AVFs other than by history alone. ${ }^{2}$

Increased venous pressure has been shown to generate AVFs in rats. ${ }^{5}$ It has been suggested that S-AVFs can become enlarged by capturing subcutaneous or intracranial feeder vessels and that the consequent hemodynamic stress may induce de novo aneurysms in S-AVFs. Additionally, intracranial AVFs may alter the intracranial hemodynamics, leading to increased venous pressure in the superior sagittal sinus and the formation of communicating S-AVFs. ${ }^{5}$ Interestingly, our patient had an intracranial AVF treated with endovascular embolization 1 year prior to the formation of the S-AVF.
An angiogram at the time of this embolization procedure did not demonstrate any S-AVFs. Furthermore, our patient has a history of 2 substantial falls in childhood with head trauma and loss of consciousness. Perhaps these traumas initiated a channel through the cranium where an S-AVF with intracranial communication was able to form and may have only become clinically or radiographically detectable once it enlarged due to the altered hemodynamics caused by the intracranial AVF 1 year prior.

The diagnosis of an S-AVF is confirmed with imaging studies. Doppler ultrasonography initially will help to detect that a lesion is vascular in nature. Intra-arterial digital subtraction angiography is the gold-standard imaging technique and is necessary to delineate the feeding arteries and the draining channels as well as possible communication with intracranial yasculature. ${ }^{1,2}$

There is controversy regarding the appropriate treatment of S-AVFs. ${ }^{2}$ Each S-AVF possesses unique anatomic features that dictate appropriate management. The prognosis for an S-AVF is extremely variable, and the decision to treat is based on the patient's symptoms and risk for exsanguinating hemorrhage., ${ }^{2,4}$ Neurosurgical approaches include ligation of the feeding arteries, surgical resection, electrothrombosis, direct intralesional injection of sclerosing agents, and endovascular embolization. Endovascular interyention increasingly is utilized as a primary treatment or as a preoperative adjunct to surgery. ${ }^{2,4}$ Large S-AVFs have a high risk for recurrence after treatment with endovascular embolization alone. In cases with intracranial communication, the intracranial component is treated first. ${ }^{2}$

This case emphasizes the importance of including $\mathrm{S}$-AVFs on the dermatologic differential diagnosis of a scalp nodule, especially in patients with any history of intracranial AVF. A thorough history, detailed intake of potential signs and symptoms of AVF, and palpation for bruits is recommended as part of the surgical evaluation of a scalp nodule. Imaging of scalp nodules also should be considered for patients with any history of intracranial AVF; S-AVFs should be referred to neurosurgery or interventional neuroradiology for evaluation and possible treatment.

\section{REFERENCES}

1. Bernstein J, Podnos S, Leavitt M. Arteriovenous fistula following hair transplantation. Dermatol Surg. 2011;37:873-875.

2. Kumar R, Sharma G, Sharma BS. Management of scalp arterio-venous malformation: case series and review of literature. $\mathrm{Br} J$ Neurosurg. 2012;26:371-377.

3. Gurkanlar D, Gonul M, Solmaz I, et al. Cirsoid aneurysms of the scalp. Neurosurg Rev. 2006;29:208-212.

4. Senoglu M, Yasim A, Gokce M, et al. Nontraumatic scalp arteriovenous fistula in an adult: technical report on an illustrative case. Surg Neurol. 2008;70:194-197.

5. Lanzino G, Passacantilli E, Lemole G, et al. Scalp arteriovenous malformation draining into the superior sagittal sinus associated with an intracranial arteriovenous malformation: just a coincidence? case report. Neurosurgery. 2003;52:440-443. 